\title{
A New Sequence and Its Some Congruence Properties
}

\section{Wenpeng Zhang and Xin Lin *}

School of Mathematics, Northwest University, Xi'an 710127, China; wpzhang@nwu.edu.cn

* Correspondence: estelle-xin@hotmail.com

Received: 17 July 2018; Accepted: 20 August 2018; Published: 24 August 2018

Abstract: The aim of this paper is to study the congruence properties of a new sequence, which is closely related to Fubini polynomials and Euler numbers, using the elementary method and the properties of the second kind Stirling numbers. As results, we obtain some interesting congruences for it. This solves a problem proposed in a published paper.

Keywords: Fubini polynomials; Euler numbers; congruence; elementary method

MSC: 11B83; 11B37

\section{Introduction}

Let $n \geq 0$ be an integer, the famous Fubini polynomials $F_{n}(y)$ are defined according to the coefficients of following generating function:

$$
\frac{1}{1-y\left(e^{t}-1\right)}=\sum_{n=0}^{\infty} \frac{F_{n}(y)}{n !} \cdot t^{n}
$$

where $F_{0}(y)=1, F_{1}(y)=y$, and so on.

These polynomials are closely related to the Stirling numbers and Euler numbers. For example, if $y=-\frac{1}{2}$, then (1) becomes

$$
\frac{2}{1+e^{t}}=\sum_{n=0}^{\infty} \frac{E_{n}}{n !} \cdot t^{n}
$$

where $E_{n}$ denotes the Euler numbers.

At the same time, the Fubini polynomials with two variables can also be defined by the following identity (see [1,2]):

$$
\frac{e^{x t}}{1-y\left(e^{t}-1\right)}=\sum_{n=0}^{\infty} \frac{F_{n}(x, y)}{n !} \cdot t^{n},
$$

and $F_{n}(y)=F_{n}(0, y)$ for all integers $n \geq 0$. Many scholars have studied the properties of $F_{n}(x, y)$, and have obtained many important works. For example, T. Kim et al. proved a series of identities related to $F_{n}(x, y)$ (see [2,3]), one of which is

$$
F_{n}(x, y)=\sum_{l=0}^{n}\left(\begin{array}{l}
n \\
l
\end{array}\right) x^{l} \cdot F_{n-l}(y), n \geq 0
$$

Zhao Jianhong and Chen Zhuoyu [4] studied the computational problem of the sums

$$
\sum_{a_{1}+a_{2}+\cdots+a_{k}=n} \frac{F_{a_{1}}(y)}{\left(a_{1}\right) !} \cdot \frac{F_{a_{2}}(y)}{\left(a_{2}\right) !} \cdots \frac{F_{a_{k}}(y)}{\left(a_{k}\right) !}
$$


where the summation in the formula above denotes all $k$-dimension non-negative integer coordinates $\left(a_{1}, a_{2}, \cdots, a_{k}\right)$ such that $a_{1}+a_{2}+\cdots+a_{k}=n$. They proved the identity

$$
\begin{aligned}
& \sum_{a_{1}+a_{2}+\cdots+a_{k}=n} \frac{F_{a_{1}}(y)}{\left(a_{1}\right) !} \cdot \frac{F_{a_{2}}(y)}{\left(a_{2}\right) !} \cdots \frac{F_{a_{k}}(y)}{\left(a_{k}\right) !} \\
= & \frac{1}{(k-1) !(y+1)^{k-1}} \cdot \frac{1}{n !} \sum_{i=0}^{k-1} C(k-1, i) F_{n+k-1-i}(y),
\end{aligned}
$$

where the sequence $C(k, i)$ is defined for positive integer $k$ and $i$ with $0 \leq i \leq k, C(k, 0)=1$, $C(k, k)=k !$ and

$$
C(k+1, i+1)=C(k, i+1)+(k+1) C(k, i), \text { for all } 0 \leq i<k,
$$

providing $C(k, i)=0$, if $i>k$.

For clarity, for $1 \leq k \leq 9$, we list values of $C(k, i)$ in the following Table 1 .

Table 1. Values of $C(k, i)$.

\begin{tabular}{ccccccccccc}
\hline$C(\boldsymbol{k}, \boldsymbol{i})$ & $\boldsymbol{i}=\mathbf{0}$ & $\boldsymbol{i = 1}$ & $\boldsymbol{i = 2}$ & $\boldsymbol{i = 3}$ & $\boldsymbol{i = 4}$ & $\boldsymbol{i = 5}$ & $\boldsymbol{i = 6}$ & $\boldsymbol{i = 7}$ & $\boldsymbol{i}=\mathbf{8}$ & $\boldsymbol{i}=\mathbf{9}$ \\
\hline $\boldsymbol{k}=\mathbf{1}$ & 1 & 1 & & & & & & & & \\
$\boldsymbol{k}=\mathbf{2}$ & 1 & 3 & 2 & & & & & & & \\
$\boldsymbol{k}=\mathbf{3}$ & 1 & 6 & 11 & 6 & & & & & & \\
$\boldsymbol{k}=\mathbf{4}$ & 1 & 10 & 35 & 50 & 24 & & & & & \\
$\boldsymbol{k}=\mathbf{5}$ & 1 & 15 & 85 & 225 & 274 & 120 & & & & \\
$\boldsymbol{k}=\mathbf{6}$ & 1 & 21 & 175 & 735 & 1624 & 1764 & 720 & & & \\
$\boldsymbol{k}=\mathbf{7}$ & 1 & 28 & 322 & 1960 & 6769 & 13,132 & 13,068 & 5040 & & \\
$\boldsymbol{k}=\mathbf{8}$ & 1 & 36 & 546 & 4536 & 22,449 & 67,284 & 118,124 & 109,584 & 40,320 & \\
$\boldsymbol{k}=\mathbf{9}$ & 1 & 45 & 870 & 9450 & 63,273 & 269,325 & 723,680 & $1,172,700$ & $1,026,576$ & 362,880 \\
\hline
\end{tabular}

Meanwhile, Zhao Jianhong and Chen Zhuoyu [4] proposed some conjectures related to the sequence. We believe that this sequence is meaningful because it satisfies some very interesting congruence properties, such as

$$
C(p-2, i) \equiv 1(\bmod p)
$$

for all odd primes $p$ and integers $0 \leq i \leq p-2$. The equivalent conclusion is

$$
C(p-1, i) \equiv 0(\bmod p)
$$

for all odd primes $p$ and positive integers $1 \leq i \leq p-2$. Since some related content can be found in references [5-15], we will not go through all of them here.

The aim of this paper is to prove congruence (5) by applying the elementary method and the properties of the second kind Stirling numbers. That is, we will solve the conjectures in [4], which are listed in the following.

Theorem 1. Let $p$ be an odd prime. For any integer $1 \leq i \leq p-2$, we have congruence

$$
C(p-1, i) \equiv 0(\bmod p) \text {. }
$$

From this theorem and (3), we can deduce following three corollaries:

Corollary 1. For any positive integer $n$ and odd prime $p$, we have

$$
F_{n+p-1}(y)-F_{n}(y) \equiv 0(\bmod p)
$$

Corollary 2. For any positive integer $n$ and odd prime $p$, we have 


$$
E_{n+p-1}-E_{n} \equiv 0(\bmod p)
$$

Corollary 3. For any odd prime $p$, we have the congruences

$$
2 E_{p} \equiv-1(\bmod p), 4 E_{p+2} \equiv 1(\bmod p) \text {, and } 2 E_{p+4} \equiv-1(\bmod p) .
$$

Note. Since $E_{n}$ is a rational number, we can denote $E_{n}=\frac{U_{n}}{V_{n}}$, where $U_{n}$ and $V_{n}$ are integers with $\left(U_{n}, V_{n}\right)=1$. Based on this, in our paper, the expression $E_{n} \equiv 0(\bmod p)$ means $p \mid U_{n}$, while $p \nmid V_{n}$.

\section{Several Lemmas}

Lemma 1. For any positive integer $k$, we have the identity

$$
k ! y(y+1)^{k-1}=\sum_{i=0}^{k-1} C(k-1, i) F_{k-i}(y) .
$$

Proof. Taking $n=1$ in (3), and noting that $F_{0}(y)=1, F_{1}(y)=y$, and the equation $a_{1}+a_{2}+\cdots+a_{k}=1$ holds if and only if one of $a_{i}$ is 1 , others are 0 . The number of the solutions of this equation is $\left(\begin{array}{l}k \\ 1\end{array}\right)=k$. So, from (3), we have

$$
\begin{aligned}
& \sum_{a_{1}+a_{2}+\cdots+a_{k}=1} \frac{F_{a_{1}}(y)}{\left(a_{1}\right) !} \cdot \frac{F_{a_{2}}(y)}{\left(a_{2}\right) !} \cdots \frac{F_{a_{k}}(y)}{\left(a_{k}\right) !}=\left(\begin{array}{l}
k \\
1
\end{array}\right) y=k y \\
= & \frac{1}{(k-1) !(y+1)^{k-1}} \cdot \sum_{i=0}^{k-1} C(k-1, i) F_{k-i}(y)
\end{aligned}
$$

or identity

$$
k ! y(y+1)^{k-1}=\sum_{i=0}^{k-1} C(k-1, i) F_{k-i}(y),
$$

which proves Lemma 1.

Lemma 2. For any positive integer $n$, we have the identity

$$
F_{n}(y)=\sum_{k=0}^{n} S(n, k) k ! y^{k},(n \geq 0)
$$

where $S(n, k)$ are the second kind Stirling numbers, which are defined for any integer $k, n$ with $0 \leq k \leq n$ as:

$$
S(n, k)=k S(n-1, k)+S(n-1, k-1)
$$

where $S(0,0)=1, S(n, 0)=0$ and $S(0, k)=0$ for $n, k>0$.

Proof. See Reference [2].

Lemma 3. For any positive integers $n$ and $k$, we have

$$
S(n, k)=\frac{1}{k !} \sum_{j=0}^{k}\left(\begin{array}{l}
k \\
j
\end{array}\right) j^{n}(-1)^{k-j} .
$$

Proof. See Theorem 4.3.12 of [16]. 
Lemma 4. For any odd prime $p$ and positive integer $2 \leq k \leq p-1$, we have the congruence

$$
k ! S(p, k) \equiv 0(\bmod p) .
$$

Proof. From the definition and properties of $S(n, k)$, we have $S(n, k)=0$, if $k>n$. For any integers $0 \leq j \leq p-1$, from the famous Fermat's little theorem, we have the congruence $j^{p} \equiv j(\bmod p)$. From this congruence and Lemma 3, we have

$$
k ! S(p, k)=\sum_{j=0}^{k}\left(\begin{array}{l}
k \\
j
\end{array}\right) j^{p}(-1)^{k-j} \equiv \sum_{j=0}^{k}\left(\begin{array}{l}
k \\
j
\end{array}\right) j(-1)^{k-j} \equiv k ! S(1, k) \equiv 0(\bmod p),
$$

if $k \geq 2$. This completes the proof of Lemma 4 .

\section{Proof of the Theorem}

In this section, we will prove Theorem by mathematical induction. Taking $k=p$ in Lemma 1 and noting that $C(p-1,0)=1$ and $C(p-1, p-1)=(p-1)$ !, we have:

$$
\begin{gathered}
p ! y(y+1)^{p-1}=\sum_{i=0}^{p-1} C(p-1, i) F_{p-i}(y) \\
=F_{p}(y)+y(p-1) !+\sum_{i=1}^{p-2} C(p-1, i) F_{p-i}(y) .
\end{gathered}
$$

Note that $(p-1) !+1 \equiv 0(\bmod p)$, which implies

$$
F_{p}(y)-y+\sum_{i=1}^{p-2} C(p-1, i) F_{p-i}(y) \equiv 0(\bmod p) .
$$

From (6), we have the congruence

$$
y-F_{p}(y) \equiv \sum_{i=1}^{p-2} C(p-1, i) F_{p-i}(y)(\bmod p)
$$

From Lemma 2, we have

$$
F_{p}(y)=\sum_{k=0}^{p} S(p, k) k ! y^{k}
$$

and

$$
F_{p}^{(p-1)}(0)=S(p, p-1)(p-1) ! \cdot(p-1) !
$$

where $F_{n}^{(k)}(y)$ denotes the $k$-order derivative of $F_{n}(y)$ for variable $y$.

$$
F_{p-1}^{(p-1)}(0)=S(p-1, p-1)(p-1) ! \cdot(p-1) !=(p-1) ! \cdot(p-1) ! .
$$

Then, applying Lemma 3 and Lemma 4 and noting that $S(1, p-1)=0$, we have

$$
\begin{aligned}
(p-1) ! S(p, p-1) & \equiv \sum_{j=0}^{p-1}\left(\begin{array}{c}
p-1 \\
j
\end{array}\right) j^{p}(-1)^{p-1-j} \equiv \sum_{j=0}^{p-1}\left(\begin{array}{c}
p-1 \\
j
\end{array}\right) j(-1)^{p-1-j} \\
& \equiv(p-1) ! S(1, p-1) \equiv 0(\bmod p) .
\end{aligned}
$$


Combining (7), (9), (10), and (11), we have:

$$
0 \equiv-S(p, p-1)(p-1) !(p-1) ! \equiv C(p-1,1)(p-1) ! \cdot(p-1) !(\bmod p)
$$

or

$$
C(p-1,1) \equiv 0(\bmod p) .
$$

That is, the theorem is true for $i=1$.

Assume that the theorem is true for all $1 \leq i \leq s$. That is,

$$
C(p-1, i) \equiv 0(\bmod p)
$$

for $1 \leq i \leq s<p-1$. It is clear that if $s=p-2$, then the theorem is true.

If $1<s<p-2$, then from (7) we have the congruence

$$
y-F_{p}(y) \equiv \sum_{i=s+1}^{p-2} C(p-1, i) F_{p-i}(y)(\bmod p) .
$$

In congruence (14), taking the $(p-s-1)$-order derivative with respect to $t$, then let $y=0$, applying Lemma 2, we have:

$$
\begin{aligned}
& -S(p, p-s-1)(p-s-1) ! \cdot(p-s-1) ! \\
\equiv & C(p-1, s+1)(p-s-1) !(p-s-1) !(\bmod p) .
\end{aligned}
$$

Note that $((p-s-1) !, p)=1$, from Lemma 4 and (15) we have the congruence

$$
C(p-1, s+1)(p-s-1) ! \equiv-(p-s-1) ! S(p, p-s-1) \equiv 0(\bmod p),
$$

which implies

$$
C(p-1, s+1) \equiv 0(\bmod p) .
$$

That is, the theorem is true for $i=s+1$. Now the proof of the theorem completes by mathematical induction.

Now, we prove Corollary 1 . For any integer $n \geq 0$, taking $k=p$ in (3) and noting that

$$
n ! \sum_{a_{1}+a_{2}+\cdots+a_{p}=n} \frac{F_{a_{1}}(y)}{\left(a_{1}\right) !} \cdot \frac{F_{a_{2}}(y)}{\left(a_{2}\right) !} \cdots \frac{F_{a_{p}}(y)}{\left(a_{p}\right) !} \equiv 0(\bmod p),
$$

we have

$$
\sum_{i=0}^{p-1} C(p-1, i) F_{n+p-1-i}(y) \equiv 0(\bmod p) .
$$

From our theorem, we have

$$
\sum_{i=1}^{p-2} C(p-1, i) F_{n+p-1-i}(y) \equiv 0(\bmod p) .
$$


Note that $C(p-1,0)=1, C(p-1, p-1)=(p-1)$ !. Combining (16) and (17), we can deduce the congruence

$$
F_{n+p-1}(y)-F_{n}(y) \equiv 0(\bmod p) .
$$

Now the proof of Corollary 1 completes. Since Corollarys 2 and 3 are the special situation of Corollary 1, we will not prove Corollarys 2 and 3 here.

Author Contributions: Conceptualization, W.Z.; Methodology, W.Z. and X.L.; Software, X.L.; Validation, W.Z. and X.L.; Formal Analysis, W.Z.; Investigation, X.L.; Resources, W.Z.; Data Curation, X.L.; Writing Original Draft Preparation, W.Z.; Writing Review \& Editing, X.L.; Visualization, W.Z.; Supervision, W.Z.; Project Administration, X.L.; Funding Acquisition, W.Z. All authors have read and approved the final manuscript.

Funding: This research was funded by [National Natural Science Foundation of China] grant number [11771351].

Acknowledgments: The authors would like to thank the reviewers for their very detailed and helpful comments, which have significantly improved the presentation of this paper.

Conflicts of Interest: The authors declare no conflict of interest.

\section{References}

1. Kilar, N.; Simsek, Y. A new family of Fubini type numbrs and polynomials associated with Apostol-Bernoulli nujmbers and polynomials. J. Korean Math. Soc. 2017, 54 , 1605-1621.

2. Kim, T.; Kim, D.S.; Jang, G.-W. A note on degenerate Fubini polynomials. Proc. Jiangjeon Math. Soc. 2017, 20, 521-531.

3. Kim, T.; Kim, D.S.; Jang, G.-W.; Kwon, J. Symmetric identities for Fubini polynomials. Symmetry 2018, 10, 219. [CrossRef]

4. Zhao, J.-H.; Chen, Z.-Y. Some symmetric identities involving Fubini polynomials and Euler numbers. Symmetry 2018, 10, 303.

5. Chen, L.; Zhang, W.-P. Chebyshev polynomials and their some interesting applications. Adv. Differ. Equ. 2017, 2017, 303.

6. Clemente, C. Identities and generating functions on Chebyshev polynomials. Georgian Math. J. 2012, 19, 427-440.

7. He, Y. Symmetric identities for Calitz's q-Bernoulli numbers and polynomials. Adv. Differ. Equ. 2013, 2013, 246. [CrossRef]

8. Kim, T. Symmetry of power sum polynomials and multivariate fermionic $p$-adic invariant integral on $Z_{p}$. Russ. J. Math. Phys. 2009, 16, 93-96. [CrossRef]

9. Kim, T.; Kim, D.S. An identity of symmetry for the degernerate Frobenius-Euler polynomials. Math. Slovaca 2018, 68, 239-243. [CrossRef]

10. Li, X.-X. Some identities involving Chebyshev polynomials. Math. Probl. Eng. 2015, 2015, 950695. [CrossRef]

11. Rim, S.-H.; Jeong, J.-H.; Lee, S.-J.; Moon, E.-J.; Jin, J.-H. On the symmetric properties for the generalized twisted Genocchi polynomials. ARS Comb. 2012, 105, 267-272.

12. Wang, T.-T.; Zhang, W.-P. Some identities involving Fibonacci, Lucas polynomials and their applications. Bull. Math. Soc. Sci. Math. Roum. 2012, 55, 95-103.

13. Yi, Y.; Zhang, W.P. Some identities involving the Fibonacci polynomials. Fibonacci Q. 2002, 40, $314-318$.

14. Zhang, W.-P. Some identities involving the Euler and the central factorial numbers. Fibonacci Q. 1998, 36, 154-157.

15. Kim, D.S.; Park, K.H. Identities of symmetry for Bernoulli polynomials arising from quotients of Volkenborn integrals invariant under $S_{3}$. Appl. Math. Comput. 2013, 219, 5096-5104. [CrossRef]

16. Feng R.-Q.; Song C.-W. Combinatorial Mathematics; Beijing University Press: Beijing, China, 2015.

(C) 2018 by the authors. Licensee MDPI, Basel, Switzerland. This article is an open access article distributed under the terms and conditions of the Creative Commons Attribution (CC BY) license (http:/ / creativecommons.org/licenses/by/4.0/). 